\title{
Emodin ameliorates acute pancreatitis-induced lung injury by suppressing NLRP3 inflammasome-mediated neutrophil recruitment
}

\author{
NAN JIANG ${ }^{1-3}$, ZHAOXIA LI $^{1,2}$, YALAN LUO $^{1,2}$, LIU JIANG $^{1,2}$, \\ GUIXIN ZHANG ${ }^{1,2}$, QI YANG ${ }^{4}$ and HAILONG CHEN ${ }^{1}$
}

\author{
${ }^{1}$ Department of General Surgery, The First Affiliated Hospital of Dalian Medical University, Dalian, Liaoning 116011; \\ ${ }^{2}$ Institute (College) of Integrative Medicine and College of Pharmacy, Dalian Medical University, Dalian, Liaoning 116044; \\ ${ }^{3}$ Department of Obstetrics and Gynecology, The First Affiliated Hospital of Dalian Medical University, Dalian, \\ Liaoning 116011; ${ }^{4}$ Department of Traditional Chinese Medicine, The Second Affiliated Hospital of \\ Dalian Medical University, Dalian, Liaoning 116027, P.R. China
}

Received June 14, 2020; Accepted March 17, 2021

DOI: $10.3892 / \mathrm{etm} .2021 .10289$

\begin{abstract}
Severe acute pancreatitis (SAP) activates the systemic inflammatory response and is potentially lethal. The aim of the present study was to determine the effects of emodin on acute lung injury (ALI) in rats with SAP and investigate the role of the Nod-like receptor protein 3 (NLRP3) inflammasome and its association with neutrophil recruitment. Sodium taurocholate $(5.0 \%)$ was used to establish the SAP model. All animals were randomly assigned into four groups: Sham, SAP, emodin and dexamethasone (positive control drug) groups ( $\mathrm{n}=10$ mice per group). Histopathology observation of pancreatic and lung tissues was detected by hematoxylin and eosin staining. The levels of serum amylase, IL-1 $\beta$ and IL-18 were measured by ELISA. Single-cell suspensions were obtained from enzymatically digested lung tissues, followed by flow cytometric analysis for apoptosis. In addition, the expression levels of NLRP3 inflammasome-associated and apoptosis-associated proteins in lung tissues were measured by western blotting. Moreover, lymphocyte antigen 6 complex locus $\mathrm{G}^{+} \mathrm{D}^{+}\left(\mathrm{Ly}_{6} \mathrm{G}^{+}\right)$cell recruitment was detected using immunohistochemical analysis. The results revealed that emodin markedly improved pancreatic histological injury
\end{abstract}

Correspondence to: Dr Qi Yang, Department of Traditional Chinese Medicine, The Second Affiliated Hospital of Dalian Medical University, 467 Zhongshan Road, Dalian, Liaoning 116027, P.R. China

E-mail: dyeyyq1016@163.com

Professor Hailong Chen, Department of General Surgery, The First Affiliated Hospital of Dalian Medical University, 222 Zhongshan Road, Dalian, Liaoning 116011, P.R. China

E-mail: chenhailong2007@yahoo.com

Key words: acute pancreatitis, acute lung injury, emodin, Nod-like receptor protein 3 , neutrophil and decreased the levels of serum amylase, IL-1 $\beta$ and IL-18. Pulmonary edema and apoptosis were significantly alleviated by emodin. Additionally, the protein expression levels of intercellular adhesion molecule 1,NLRP3, apoptosis-associated speck-like protein containing a CARD and cleaved caspase-1 were downregulated following emodin treatment. Moreover, emodin inhibited $\mathrm{Ly} 6 \mathrm{G}^{+}$cell recruitment in lung tissues. The present study demonstrated that emodin may offer protection against ALI induced by SAP via inhibiting and suppressing NLRP3 inflammasome-mediated neutrophil recruitment and may be a novel therapeutic strategy for the clinical treatment of ALI.

\section{Introduction}

Acute pancreatitis (AP) is a common clinical condition, with an increasing incidence over recent years (1). For example, the hospital admissions for AP doubled in the English population between 1963 and 1998 (2). Irrespective of the cause, activation of digestive enzymes within pancreatic acinar cells is thought to be a critical event, which can initiate and propagate pancreatic damage during AP (3). In addition to pancreatic injury, the pathophysiology of severe AP (SAP) involves a systemic inflammatory response, often resulting in distant organ dysfunction (4). Acute lung injury (ALI) is the most common distant organ disease in SAP and is the main cause of SAP-associated death (5). The pathological mechanism of SAP-ALI is very complex and has not been fully elucidated. Proinflammatory cytokines secreted by activated inflammatory cells have been implicated in the propagation and amplification of the systemic inflammatory response in SAP (6). Presently, the detrimental effects of the excessive inflammatory cascade in the pathogenesis of ALI associated with SAP have been extensively studied (7-9). However, the initiating mechanisms of inflammation in the lungs are not well defined.

Previous studies have demonstrated that the inflammatory cascade induced by sterile inflammasome activation is a 
major component in a wide range of diseases, including SAP and ALI $(10,11)$. Molecular signaling pathways that have been identified to contribute to sterile inflammation include toll-like receptor (TLR) signaling pathways, specific inflammasome complexes and IL-1 signaling pathways (12). TLR4 is an important mediator of the inflammatory response in SAP (13-15). Additionally, baicalin targeting TLR4 for ALI induced by AP has been mentioned (6). IL-1 $\beta$ is closely associated with SAP, and its increase is positively correlated with disease severity (16). The pathological damage of the pancreas, the degree of inflammation and the severity of SAP-ALI are significantly decreased after blocking IL-1 $\beta$ expression in SAP (8). Nod-like receptor protein 3 (NLRP3), an intracellular recognition receptor, is involved in homotypic interactions and works with an adaptor protein known as apoptosis-associated speck-like protein containing a CARD (ASC) when triggered by a range of stimuli, including infection, tissue damage and metabolic dysregulation (17). NLRP3 continues to recruit caspase-1 and forms an intracellular multiprotein complex known as an inflammasome (18). Recent studies have clarified the role of NLRP3 in the development of AP or ALI $(8,19)$. Aggregation of the NLRP3 inflammasome stimulates caspase- 1 and facilitates the processing and the secretion of the proinflammatory cytokine IL-1 $\beta$, which serves an important part in inflammatory responses (20). Particularly, suppression of NLRP3 decreases neutrophil recruitment (21). Notably, the long-held belief that neutrophils recruited to tissue injury are terminally activated to fulfill their roles of pathogen defense, bystander tissue damage and/or tissue repair has been challenged (22). There is emerging evidence that neutrophils may exist in various subtypes, may have multifaceted roles and may possess intermediate stages of activation even after leaving the circulation (23). To the best of our knowledge, the activation state and function of recruited neutrophils in ALI have not been studied.

Emodin (1,3,8-trihydroxy-6-methylemodin) is an active constituent of oriental medicinal herbs, including Rheum officinale (24). Emodin significantly eases pulmonary edema and improves SAP-induced ALI $(25,26)$. A number of studies investigating the anti-inflammatory potential of emodin have also been reported $(27,28)$. However, the functional involvement of emodin in neutrophil recruitment and IL-1 $\beta$ secretion through inflammasome regulation has not been reported in SAP-induced ALI in vivo (29). On the other hand, dexamethasone (DEX) is an important glucocorticoid that is vital in anti-inflammation and anti-toxicity, and can be used to alleviate early inflammatory reactions in the lungs in SAP (30). Several studies have provided experimental basis for the protective role of DEX in lung inflammation by targeting the NLRP3 inflammasome signaling pathway $(31,32)$. The present study aimed to investigate the functional capacity of emodin in the improvement of inflammation in the lungs induced by SAP and to evaluate the role of NLRP3 inflammasomes to provide further information regarding the clinical treatment options for ALI.

\section{Materials and methods}

Animals. A total of 40 male 6-week-old Wistar rats weighing 200-250 g were purchased from the Animal Center of Dalian
Medical University (Dalian, China). The Institutional Animal Care and Use Committee of Dalian Medical University approved the protocols for all the experiments (approval no. AEE19003), which were performed in the laboratory of the Animal Center of Dalian Medical University under the approved protocols.

Experimental process. All rats were given free food and water and kept under standard conditions (room temperature, $22 \pm 2^{\circ} \mathrm{C}$; 12-h light/dark cycle; relative humidity, 50-60\%) and left to acclimatize for 1 week. The mice were randomly assigned to the following groups: i) Sham group; ii) SAP group; iii) SAP-emodin (Emodin) group; and iv) SAP-DEX (DEX) group, with 10 rats in each group. Pentobarbital $(4 \% ; 30-40 \mathrm{mg} / \mathrm{kg})$ was injected intraperitoneally in all rats for anesthesia. Retrograde infusion treatment of 5\% sodium taurocholate ( $1 \mathrm{ml} / \mathrm{kg}$; Sigma-Aldrich; Merck $\mathrm{KGaA}$ ) into the biliary pancreatic duct was performed to induce SAP-associated ALI in the SAP, Emodin and DEX groups. Emodin (cat. no. E8390; Beijing Solarbio Science \& Technology Co., Ltd.) treatment ( $4 \mathrm{mg} / \mathrm{ml} ; 40 \mathrm{mg} / \mathrm{kg}$ ) was administered to rats via gavage $2 \mathrm{~h}$ after rats gained consciousness following SAP induction, as previously described (30). DEX (Henan Lingrui Pharmaceutical Co., Ltd.) treatment $(5 \mathrm{mg} / \mathrm{ml} ; 2 \mathrm{ml} / \mathrm{kg}$ ) was intraperitoneally injected at $2 \mathrm{~h}$ post-SAP induction in the DEX group as previously described (33). Rats were anesthetized using pentobarbital $(40 \mathrm{mg} / \mathrm{kg}$ ) intraperitoneally before euthanasia by exsanguination at $24 \mathrm{~h}$ post-modeling. Blood and lung tissues were collected and stored at $4^{\circ} \mathrm{C}$ and $-80^{\circ} \mathrm{C}$, respectively, for analysis.

Preparation of single-cell suspensions of lung and flow cytometric analysis of apoptosis. After the rats were sacrificed, their lungs were perfused through the right ventricle with $5 \mathrm{ml}$ PBS. The lungs were removed and the large airways were dissected from the peripheral lung tissue. The peripheral lung tissue was cut into small pieces with scissors, transferred into C-tubes (Miltenyi Biotec, Inc.) and processed in digestion buffer $(1 \mathrm{mg} / \mathrm{ml}$ of Collagenase D and $0.1 \mathrm{mg} / \mathrm{ml}$ DNase I; both dissolved in Hanks' balanced salt solution; both from Roche Diagnostics) and a GentleMACS dissociator (Miltenyi Biotec $\mathrm{GmbH}$ ), according to the manufacturer's instructions. Homogenized lungs were passed through a $40-\mu$ m nylon mesh to obtain a single-cell suspension. The remaining red blood cells were lysed using BD Pharm Lyse (BD Biosciences). The resultant cells were counted using a Countess cell counter (Invitrogen; Thermo Fisher Scientific, Inc.). For apoptosis analysis, cells were stained using an Annexin V/FITC and PI apoptosis detection kit (Nanjing KeyGen Biotech Co., Ltd.). Subsequently, apoptosis was detected by flow cytometry. Briefly, cells were transferred and suspended in Annexin-binding buffer $\left(1 \times 10^{6}\right.$ cells $\left./ \mathrm{ml}\right)$, and were then incubated with Annexin V-FITC and PI for $15 \mathrm{~min}$ at room temperature in the dark and immediately detected using FACSCanto II (BD Biosciences) and analyzed with FlowJo v10 software (FlowJo LLC).

Histopathological analysis. Histopathological analysis was performed in accordance with a previously published method (29). Briefly, tissue samples obtained from the right upper lobe and from the pancreas were fixed in $4 \%$ paraformaldehyde 
solution for $24 \mathrm{~h}$ at room temperature and then embedded in paraffin. The tissues were sliced into $5-\mu \mathrm{m}$-thick sections and subjected to hematoxylin and eosin (H\&E) staining for $5 \mathrm{~min}$ at room temperature (29). Histopathological assessment of the pancreas was performed as previously described (34). The stained sections were observed using a light microscope (Olympus Corporation) at x200 magnification and edema, acinar necrosis, leukocyte infiltration, hemorrhage, fat necrosis and perivascular inflammation were scored using a scale of 0-3 (with 0 representing no histological abnormality, 1 representing slight histological abnormality, 2 representing intermediate histological abnormality, and 3 representing severe morphological deterioration) were also assessed. The overall score was the sum of the scores of edema, acinar necrosis, leukocyte infiltration, hemorrhage, fat necrosis and perivascular inflammation. Assessment of the lung injury was performed by a researcher blinded to the groups using a modified histological scoring system as described previously (35). The pathological score was assessed on a scale of 0-4, averaging the score of the following items: i) Alveolar congestion; ii) thickness of the alveolar wall; iii) aggregation of neutrophils or leukocyte infiltration in the vessel wall or air space; and iv) hemorrhage. The scores were represented as follows: 0 for normal lungs; 1 for mild $(<25 \%)$ lung involvement; 2 for moderate $(25-50 \%)$ lung involvement; 3 for severe (51-75\%) lung involvement and 4 for extremely severe $(>75 \%)$ lung involvement. The overall score obtained was based on the addition of all the scores and presented as the mean \pm standard deviation (with three sections from each lung using eight lungs per group).

$\alpha$-amylase (AMY), IL-1 $\beta$ and IL-18 analyses. All blood samples were collected through the posterior vena cava. After centrifugation at $1,500 \mathrm{x}$ for $10 \mathrm{~min}$ at $4^{\circ} \mathrm{C}$, the supernatants were collected and stored at $-80^{\circ} \mathrm{C}$. Serum levels of AMY, IL-1 $\beta$ and IL-18 were quantified using specific Rat AMY2 ELISA kit (cat. no. E-EL-R2545c), Rat IL-18 ELISA kit (cat. no. E-EL-R0567c), Rat IL-1 $\beta$ ELISA kit (cat. no. E-EL-R0012c; all from Elabscience, Inc.) according to the manufacturer's protocols. All samples were run in triplicate.

Immunohistochemical staining. Tissue samples were fixed in $4 \%$ paraformaldehyde solution for $24 \mathrm{~h}$ at room temperature and then embedded in paraffin. Sections $(10-\mu$ m-thick) of rat lungs were mounted on slides coated with poly-L-lysine. Slides were deparaffinized using xylene and rehydrated using graded percentages of ethanol. Citrate buffer $(0.01 \mathrm{~mol} / 1$ citric acid, $\mathrm{pH}$ 6.0) was used to pretreat sections for $20 \mathrm{~min}$ at $95^{\circ} \mathrm{C}$. The slides were immersed in PBS containing $3 \% \mathrm{H}_{2} \mathrm{O}_{2}$ for $10 \mathrm{~min}$ at room temperature. Tissue sections were also blocked with 10\% normal goat serum (Wuhan Servicebio Technology Co., Ltd.; cat. no. G1208) in PBS for $30 \mathrm{~min}$ at room temperature and incubated with rabbit polyclonal anti-lymphocyte antigen 6 complex locus G6D (Ly6G) antibody (Wuhan Servicebio Technology Co., Ltd.; cat. no. GB11229; $1: 100)$ at $4{ }^{\circ} \mathrm{C}$ overnight. Subsequently, sections were washed with PBS, incubated with biotinylated goat anti-rabbit IgG secondary antibody for $20 \mathrm{~min}$ at room temperature and treated with 3,3'-diaminobenzidine chromogen for $5 \mathrm{~min}$ at room temperature. Sections were counterstained using hematoxylin for $2 \mathrm{~min}$ at room temperature and observed using a light microscope at x200 magnification, and finally measured using a quantitative digital image analysis system (Image-Pro Plus 6.0; Media Cybernetics, Inc.) for semi-quantitative analysis. The slides were analyzed by a researcher who was blinded to the groups of the study.

Western blot analysis. Tissue samples obtained from rat lungs were subjected to protein extraction using a protein extraction kit (cat. no. KGP150; Nanjing KeyGen Biotech Co., Ltd.) according to the manufacturer's protocol. Protein concentrations were estimated using the bicinchoninic acid procedure (Beijing Solarbio Science \& Technology Co., Ltd.). Bovine serum albumin (Beyotime Institute of Biotechnology; cat. no. ST025) was used as the standard. Extracted proteins $(20 \mu \mathrm{g})$ were resuspended in the electrophoresis sample buffer containing $\beta$-mercaptoethanol. Protein separation was preformed via 10\% SDS-PAGE (Bio-Rad Laboratories, Inc.) and subsequently electrotransferred onto polyvinylidene fluoride membranes (EMD Millipore). Membranes were blocked using $5 \%$ skimmed milk in TBS with $0.1 \%$ Tween-20 (TBS-T) for $2 \mathrm{~h}$ at $37^{\circ} \mathrm{C}$. $\beta$-actin and GAPDH were used as the loading controls. The membranes were incubated overnight at $4^{\circ} \mathrm{C}$ with the following primary antibodies against: Cleaved caspase-3 (1:1,000; cat. no. 9661S; Cell Signaling Technology, Inc.), Bax (cat. no. 50599-2-Ig; ProteinTech Group, Inc.), Bcl2 (1:1,000; cat. no. 26593-1-AP; ProteinTech Group, Inc.), NLRP3 (1:1,000; cat. no. 19771-1-AP; ProteinTech Group, Inc.), ASC (1:1,000; cat. no. ab175449; Abcam), cleaved caspase-1 (1:1,000; cat. no. 89332S; Cell Signaling Technology, Inc.), intercellular adhesion molecule (ICAM-1; 1:1,000; cat. no. ab171123; Abcam), GAPDH (1:1,000; cat. no. 5174T; Cell Signaling Technology,Inc.) and $\beta$-actin (1:500; cat. no. AF5003; Beyotime Institute of Biotechnology). The blots were then washed with TBS-T and incubated with HRP-conjugated goat anti-rabbit (1:1,000; cat. no. sc-2004), mouse anti-goat (1:1,000; cat. no. sc-2354) or goat anti-mouse IgG secondary antibody (1:1,000; cat. no. sc-2031; all from Santa Cruz Biotechnology, Inc.) for $1 \mathrm{~h}$ at room temperature. Subsequently, the blots were extensively washed with TBS-T and exposed to ECL-plus reagent (Beyotime Institute of Biotechnology) according to the manufacturer's protocol. The light emitted was analyzed on a BioSpectrum-410 multispectral imaging system with a Chemi HR camera 410 (Bio-Rad Laboratories, Inc.). Under the transmitted ultraviolet light, protein expression was visualized as bands and photographed. The images were analyzed semi-quantitatively based on band densitometry using Image Lab 4.0 (Bio-Rad Laboratories, Inc.).

Statistical analysis. SPSS 20 (IBM Corp.) software package was used for all statistical analyses. Data were expressed as the mean \pm SD from three independent experiments. Data comparison among multiple groups was performed using one-way ANOVA followed by Tukey's post hoc test to determine statistically significant differences. $\mathrm{P}<0.05$ was considered to indicate a statistically significant difference.

\section{Results}

Emodin decreases the severity of SAP-induced pancreatic and lung injury. To determine the severity of SAP-induced 
pancreatic and lung injury, the serum levels of AMY were measured. The SAP group exhibited significantly increased AMY serum levels compared with the Sham group, which could be reduced by treatment with emodin or DEX (Fig. 1A). Using H\&E staining to analyze the pancreas, it was found that acinar necrosis, inflammation, hemorrhage and edema occurred in SAP rats, but these pathologies were not present in the Sham group (Fig. 1B). Furthermore, the histopathological score of the pancreas in the SAP group was significantly higher compared with that in the Sham group (Fig. 1C). These results confirmed that the SAP model was successfully established in rats. Similarly, the lung tissues of rats in the SAP group exhibited apparent alveolar wall fracture, alveolar cavity expansion, alveolar congestion and leukocyte infiltration, which is in contrast to the changes in the Sham group (Fig. 1D). The overall lung injury scores were significantly higher in the SAP group compared with in the Sham group (Fig. 1E). These results further confirmed the establishment of SAP-induced lung injury. Treatment with emodin and DEX markedly decreased the injury in pancreatic and lung tissues compared with the SAP group (Fig. 1B and D). Additionally, there was a significant decrease in the histopathological scores in emodin or DEX group, compared with the SAP group (Fig. 1C and E). These results indicated that emodin and DEX had relieving effects on ALI.

Emodin and DEX decrease apoptosis in the lungs during SAP progression. To further investigate the role of apoptosis on ALI progression, flow cytometric analysis of apoptosis was performed in each group (Fig. 2A). Compared with the Sham group, the number of apoptotic cells was significantly higher in the SAP group (Fig. 2A and B). However, emodin and DEX treatment significantly decreased the number of apoptotic cells compared with the SAP group (Fig. 2A and B). In addition, the expression levels of caspase-3, $\mathrm{Bax}$ and $\mathrm{Bcl} 2$ were evaluated. Western blotting results revealed significantly increased expression levels of cleaved caspase-3 and Bax, but a significant decrease in $\mathrm{Bcl}-2$ expression in the SAP group compared with in the Sham group (Figs. 2C-F and S1). However, in emodin- and DEX-treated rats, these changes were significantly reversed (Figs. 2C-F and S1).

Emodin decreases the activation of the NLRP3 inflammasome and suppresses neutrophil recruitment in lung tissues. Analysis of serum samples revealed significantly increased levels of IL-1 $\beta$ and IL-18 in the SAP group compared with in the Sham group (Fig. 3A and B). Meanwhile, treatment with emodin and DEX significantly decreased the levels of IL-1 $\beta$ (Fig. 3A) and IL-18 (Fig. 3B) compared with the SAP group. NLRP3 inflammasome is likely activated through several types of tissue injury or pathogen-associated signatures, with outcomes such as the autocatalytic cleavage of caspase-1, which ultimately leads to the processing and production of pro-inflammatory cytokines, particularly IL-1 $\beta$ (36). Hence, western blotting was performed to determine NLRP3 expression. The results revealed a significant upregulation in the expression levels of cleaved caspase-1, NLRP3 and ASC in the SAP group compared with in the Sham group (Figs. 3G-J and S2). Both emodin and DEX significantly inhibited this inflammatory response (Figs. 3G-J and S2). Similarly, ICAM-1 expression was significantly higher in the SAP group compared with in the Sham group, and was then significantly decreased by emodin or DEX treatment (Figs. 3C, D and S3). $\mathrm{Ly}_{6 \mathrm{G}}{ }^{+}$cell recruitment in the lungs, which is associated with neutrophil levels (37), was also measured, since neutrophils have been reported to serve an important role in lung injury (38). Immunohistochemical staining results indicated that the number of $\mathrm{Ly}_{6 \mathrm{G}}{ }^{+}$cells was significantly increased in the SAP group compared with in the Sham group, and this was then significantly decreased by emodin or DEX treatment (Fig. 3E and F).

\section{Discussion}

ALI is one of the most frequently observed diseases among patients with SAP, presenting with severe systemic complications, such as significant pulmonary edema, inflammatory infiltration in the alveoli and hyperemia (39). Understanding the mechanisms underlying SAP-induced ALI is important given the vital physiological role of this organ system and its position as an interface between the host and the environment. The respiratory system is extremely susceptible to injury and can threaten the life of an individual even with temporary functional impairment. It is reported that $20 \%$ of AP cases could lead to systemic inflammatory response syndrome and multisystem organ injury (40). The excessive generation and release of multiple inflammatory cytokines are considered as the pathogenesis of SAP-induced ALI (41). Therefore, agents with anti-inflammatory activity may be beneficial for the treatment of SAP-induced ALI and may decrease the mortality rate of patients with SAP.

Emodin or rhubarb is used as a traditional Chinese medicine and has a long history of use as an anti-inflammatory drug. Numerous studies have reported that emodin treatment can significantly decrease the production of serum AMY and inflammatory mediators or cytokines in lung tissues $(27,40)$. Additionally, DEX has been shown to act as a non-specific immune inhibitor (42). It was reported that its use inhibited the production of numerous inflammatory mediators and increased the synthesis of proteins associated with anti-inflammatory responses (43). Its use for therapeutic intervention has been reported in SAP-associated ALI (44). However, DEX treatment gives rise to some adverse effects in patients, including fungal infection, hyperglycemia, sleep insomnia and rapid weight gain (45).

The results of the present study revealed serious lung injury occurring in SAP rats compared with in Sham rats. Characteristically, more severe pulmonary edema and histopathological alterations were accompanied by increased apoptosis and inflammatory mediator production. Treatment with emodin or DEX significantly alleviated injury in lung and pancreatic tissues and suppressed inflammation. Notably, treatment with emodin exhibited improved protective effects on lung injury caused by AP compared with DEX. Therefore, the present results suggested that emodin may be a better alternative to DEX with fewer side effects.

The transmigration of neutrophils through endothelial cells serves important roles in the acute phase of pulmonary inflammation (46). Activated neutrophils are attracted by pro-inflammatory cytokines to the site of injury; subsequently, neutrophils react with excessive pro-inflammatory cytokine 

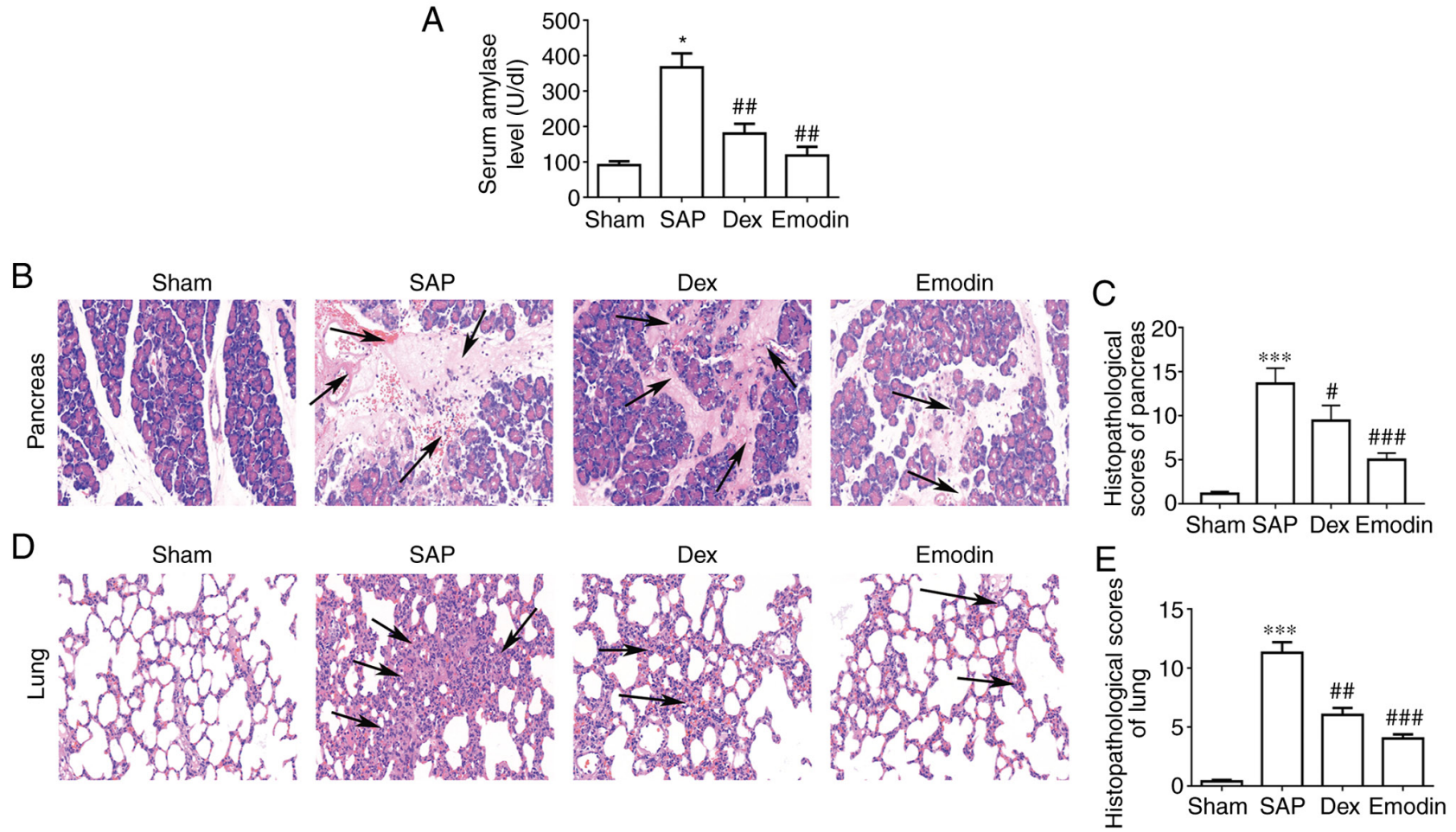

Figure 1. Emodin decreases the severity of acute pancreatitis-induced pancreatic and lung injury. (A) Serum amylase levels in each group. (B and D) Hematoxylin and eosin staining results of the pancreas and lung sections in each group taken $24 \mathrm{~h}$ after induction (magnification, x200). Histopathological scores of the (C) pancreas and (E) lungs. Data are expressed as the mean $\pm \mathrm{SD} .{ }^{*} \mathrm{P}<0.05$ and ${ }^{* * * *} \mathrm{P}<0.001$ vs. Sham; ${ }^{\#} \mathrm{P}<0.05,{ }^{\# \#} \mathrm{P}<0.01$ and

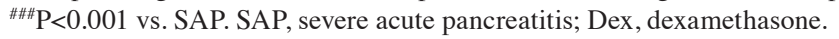

A

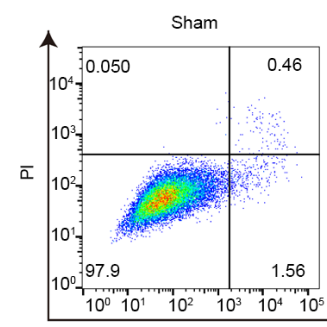

C

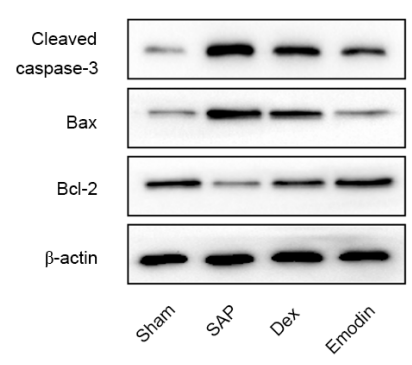

D

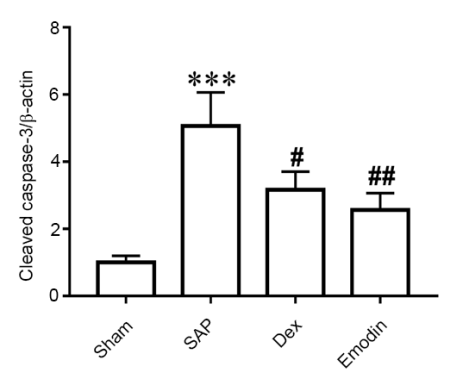

E

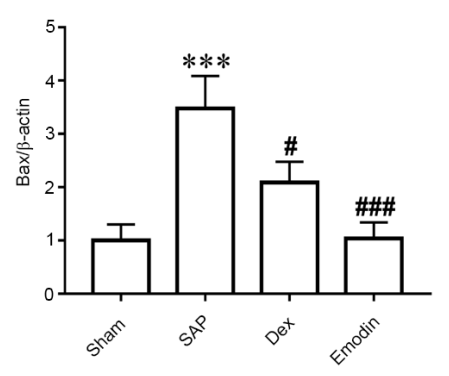

$\mathrm{B}$

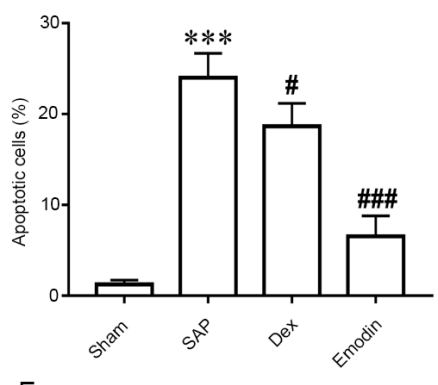

$\mathrm{F}$

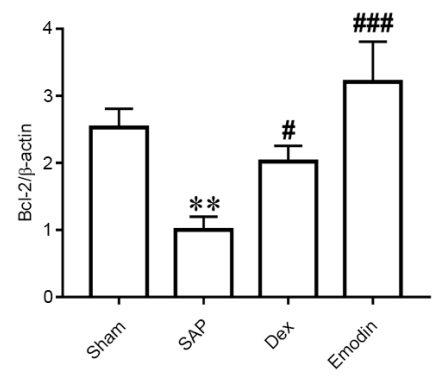

Figure 2. Emodin decreases apoptosis in the lungs during SAP progression. (A) Lung tissues were collected from each group $24 \mathrm{~h}$ after SAP modeling. Single-cell suspensions were prepared and checked for apoptosis using flow cytometry. (B) Quantification of apoptosis in the lung cells in each group. (C) Protein expression levels of (D) cleaved caspase-3, (E) Bax and (F) Bcl2 were evaluated using western blotting. Data are expressed as the mean \pm SD. ${ }^{* *} \mathrm{P}<0.01$ and ${ }^{* * *} \mathrm{P}<0.001$ vs. Sham; ${ }^{\#} \mathrm{P}<0.05,{ }^{\# \#} \mathrm{P}<0.01$ and ${ }^{\# \# \#} \mathrm{P}<0.001$ vs. SAP. SAP, severe acute pancreatitis; Dex, dexamethasone.

release and oxidative burst, which in turn further aggravates the overall cellular inflammatory response and lung tissue injury (47). Conversely, a decrease of neutrophil transmigration has been described to limit lung injury (47). The present study reported that neutrophil infiltration was attenuated after treatment with emodin, suggesting a protective role of emodin in ALI. This suggested that neutrophils may be important for ALI management. 
A

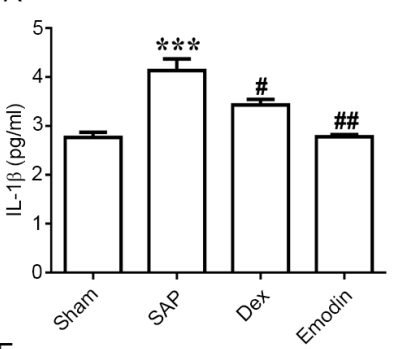

$\mathrm{E}$

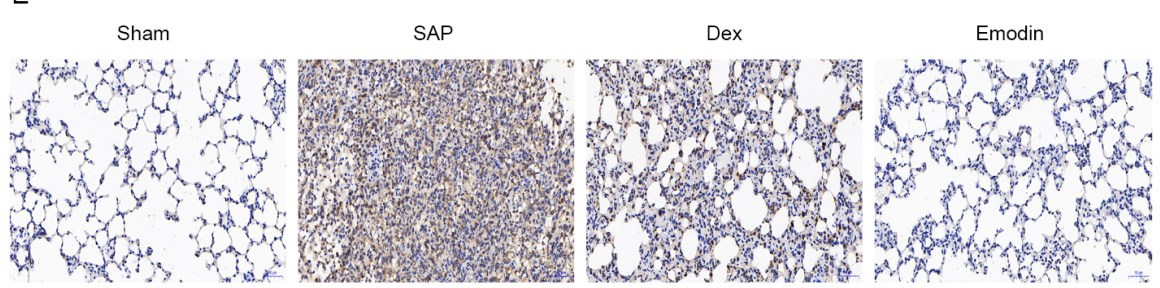

G

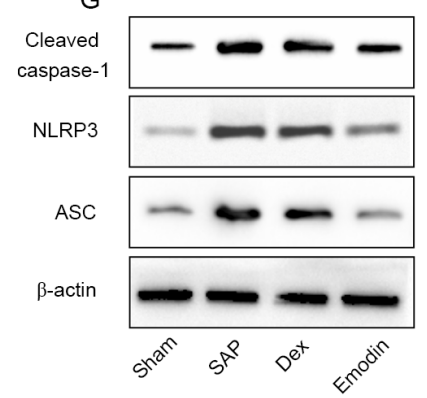

B

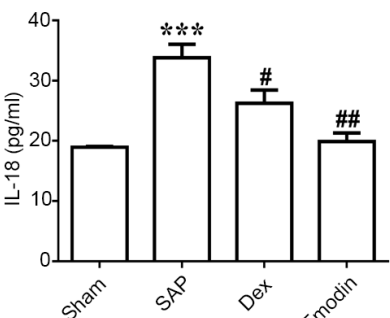

$\mathrm{H}$

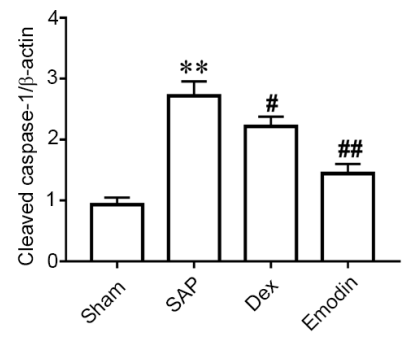

C

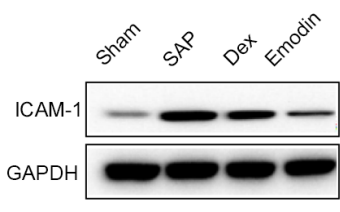

I

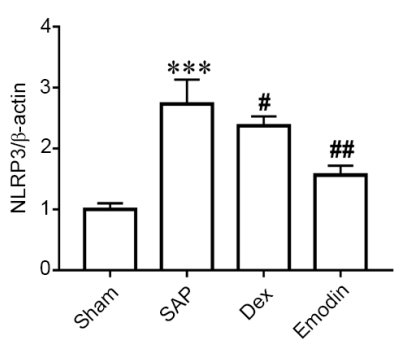

$\mathrm{D}$

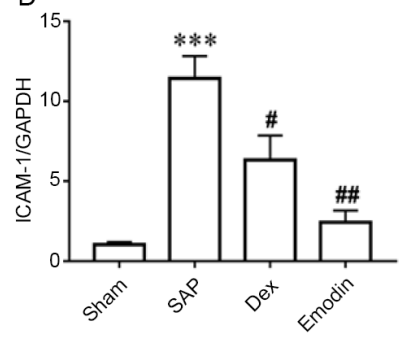

$\mathrm{F}$

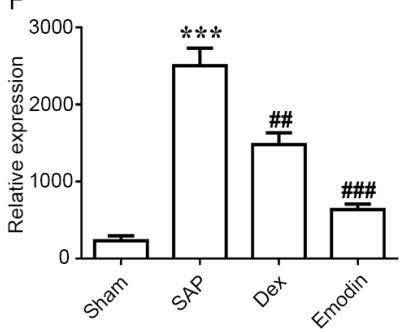

$\mathrm{J}$

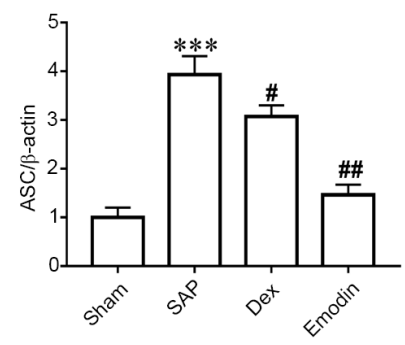

Figure 3. Emodin decreases the activation of the NLRP3 inflammasome and suppresses neutrophil recruitment in lung tissues. Serum levels of (A) IL-1 $\beta$ and (B) IL-18 were quantified using ELISA. (C and D) Protein expression levels of ICAM-1. (E and F) Immunohistochemical localization of Ly6G ${ }^{+}$cells in each group (magnification, $\mathrm{x} 200$ ). $\mathrm{Ly}_{6 \mathrm{G}}{ }^{+}$cells were positively stained in brown. The bar graph shows the relative number of Ly6G $\mathrm{G}^{+}$cells. (G-J) Protein expression levels of NLRP3 inflammasome-associated proteins in lung tissues were evaluated by western blotting. Lungs were collected from each group $24 \mathrm{~h}$ after SAP modeling. Data are expressed as the mean $\pm \mathrm{SD}$. ${ }^{* * *} \mathrm{P}<0.01$ and ${ }^{* * * *} \mathrm{P}<0.001$ vs. Sham; ${ }^{*} \mathrm{P}<0.05,{ }^{\# \#} \mathrm{P}<0.01$ and ${ }^{\# \# \#} \mathrm{P}<0.001$ vs. SAP. NLRP3, Nod-like receptor protein 3; ASC, apoptosis-associated speck-like protein containing a CARD; SAP, severe acute pancreatitis; Dex, dexamethasone; Ly6G, lymphocyte antigen 6 complex locus G6D; ICAM-1, intercellular adhesion molecule 1.

However, the molecular mechanism involving neutrophil recruitment into the lungs remains unclear. A number of studies have indicated that NLRP3 serves a key role in neutrophil recruitment, which has been demonstrated in different experimental models. For example, a murine model of gout revealed that NLRP3 was capable of mediating neutrophil recruitment (48). In another mice model where hepatic ischemia/reperfusion injury was induced, NLRP3 was reported to regulate chemokine-mediated roles and neutrophil recruitment, contributing to liver injury (49). The NLRP3 inflammasome improved the response to inflammation by promoting neutrophil infiltration (21). A previous study by Gao et al (29) reported that emodin could alleviate acute pancreatitis-associated lung injury by inhibiting NLPR3 inflammasome activation via Nrf2/HO-1 signalling. In line with the aforementioned study, the present study reported that emodin treatment inhibited NLRP3 and decreased neutrophil recruitment, thereby contributing to the protective effects of emodin treatment on ALI in a SAP model. Furthermore, the present study further demonstrated that suppression of apoptosis was involved in the benefical effect of emodin.

Although the present study did not fully examine the mechanisms by which NLRP3 initiates neutrophil recruitment into the lungs, it was speculated that the IL- $1 \beta$ signaling pathway may be potentially involved. An important role of NLRP3 is cleaving IL-1 $\beta$ into its active secretable forms (50). IL-1 $\beta$ and IL-1-receptor (IL-1R) signaling is important for triggering the expression of adhesion molecules, which are required for neutrophil attachment to vascular endothelial cells for further infiltration (51). Recently, studies have demonstrated that the IL- $1 \beta$ signaling pathway is involved in initiating neutrophil recruitment in infectious inflammation, as well as initiating neutrophil recruitment in response to sterile inflammation $(52,53)$. In a focal hepatic necrosis mice model, mice that were administered with IL- $1 \beta$ blocking antibodies exhibited a similar decrease in neutrophil accumulation (54). In addition, the number of neutrophils induced to the necrotic areas was significantly decreased in NLRP3-deficient mice compared with in wild-type mice (54). These observations suggested the potential involvement of the IL-1 $\beta$ signaling pathway in NLRP3-induced neutrophil recruitment. However, whether the IL-1 $\beta$ signaling pathway is also involved in ALI induced by AP requires further investigation. The results of the present study revealed that IL-1 $\beta$ serum levels and ICAM-1 expression in the lung were significantly increased following SAP. However, following emodin or DEX administration, a significant decrease in the IL-1 $\beta$ and ICAM-1 levels was observed, together with 
decreases in neutrophil infiltration. Overall, IL-1 $\beta$ may be one of the signaling pathways downstream of NLRP3, and further research is required to determine the involvement of the IL-1 $\beta$ signaling pathway in recruiting NLRP3-induced neutrophils. A limitation of the present study was that only male rats were used and not female rats. The reason for this was to maintain consistency with our previous studies as well as with other studies $(29,55)$. However, we plan to undertake similar experiments in female rats in future experiments to ascertain whether the effect is similar.

In conclusion, the present study demonstrated that emodin and DEX treatment prevented lung and pancreatic injury in a rat model of SAP-induced ALI. Inhibition of NLRP3 inflammasome and IL-1 $\beta$ seems to be an important factor in preventing neutrophil recruitment in the lungs. The current findings supported the notion of NLRP3 inflammasome-induced neutrophil recruitment, which may be a potential target for preventing SAP-induced ALI. Moreover, the present study suggested that emodin may be used as an alternative agent to DEX for SAP-ALI treatment.

\section{Acknowledgements}

Not applicable.

\section{Funding}

The present study was supported by the National Natural Science Fund of China (grant no. 81573751) and the Natural Science Foundation Guidance Plan of Liaoning Province (grant no. 2019-ZD-0919).

\section{Availability of data and materials}

The datasets used and/or analyzed during the current study are available from the corresponding author on reasonable request.

\section{Authors' contributions}

HC, QY and NJ conceived and designed the experiments. NJ, ZL, YL and LJ established the animal model and performed sample collection and validation experiments. NJ, QY and GZ analyzed the data and drafted the manuscript. HC edited the manuscript. HC and GZ secured funding for the study. NJ and HC confirmed the authenticity of all the raw data. All authors critically reviewed, read and approved the final manuscript.

\section{Ethics approval and consent to participate}

The animal experiments in the present study were approved by the Institutional Animal Care and Use Committee of Dalian Medical University (Dalian, China; approval no. AEE19003).

\section{Patient consent for publication}

Not applicable.

\section{Competing interests}

The authors declare that they have no competing interests.

\section{References}

1. Donnelly PE and Winch DE: Acute pancreatitis. N Engl J Med 376: 597, 2017.

2. Goldacre MJ and Roberts SE: Hospital admission for acute pancreatitis in an English population, 1963-98: Database study of incidence and mortality. BMJ 328: 1466-1469, 2004.

3. Grupp K, Bonk S, Poppe A, Wodack K, Reeh M, Gocht A, Mann O, Izbicki JR and Bachmann K: Cholecystokinin-8 treatment reduces acinar necrosis and edema of pigs with induced pancreatitis. Asian J Surg 43: 272-277, 2020.

4. Granger J and Remick D: Acute pancreatitis: Models, markers, and mediators. Shock 24 (Suppl 1): S45-S51, 2005.

5. Pastor CM, Matthay MA and Frossard JL: Pancreatitis-associated acute lung injury: New insights. Chest 124: 2341-2351, 2003.

6. Li Z, Xia X, Zhang S, Zhang A, Bo W and Zhou R: Up-regulation of Toll-like receptor 4 was suppressed by emodin and baicalin in the setting of acute pancreatitis. Biomed Pharmacother 63: 120-128, 2009.

7. Gukovskaya AS, Gukovsky I, Algül H and Habtezion A: Autophagy, inflammation, and immune dysfunction in the pathogenesis of pancreatitis. Gastroenterology 153: 1212-1226, 2017.

8. Fu Q, Zhai Z, Wang Y, Xu L, Jia P, Xia P, Liu C, Zhang X, Qin T and Zhang H: NLRP3 deficiency alleviates severe acute pancreatitis and pancreatitis-associated lung injury in a mouse model. Biomed Res Int 2018: 1294951, 2018.

9. Yang M, Chen XM, Du XG, Cao FF, Vijaya Luxmi S and Shen Q: Continuous blood purification ameliorates endothelial hyperpermeability in SAP patients with MODS by regulating tight junction proteins via ROCK. Int J Artif Organs 36: 700-709, 2013.

10. Tian X, Sun H, Casbon AJ, Lim E, Francis KP, Hellman J and Prakash A: NLRP3 inflammasome mediates dormant neutrophil recruitment following sterile lung injury and protects against subsequent bacterial pneumonia in mice. Front Immunol 8: 1337, 2017.

11. Hoque R, Farooq A, Ghani A, Gorelick F and Mehal WZ: Lactate reduces liver and pancreatic injury in Toll-like receptorand inflammasome-mediated inflammation via GPR81-mediated suppression of innate immunity. Gastroenterology 146: 1763-1774, 2014.

12. Dolinay T, Kim YS, Howrylak J, Hunninghake GM, An $\mathrm{CH}$, Fredenburgh L, Massaro AF, Rogers A, Gazourian L, Nakahira K, et al: Inflammasome-regulated cytokines are critical mediators of acute lung injury. Am J Respir Crit Care Med 185: 1225-1234, 2012.

13. Johnson GB, Brunn GJ and Platt JL: Cutting edge: An endogenous pathway to systemic inflammatory response syndrome (SIRS)-like reactions through Toll-like receptor 4. J Immunol 172: 20-24, 2004.

14. Hietaranta A, Mustonen H, Puolakkainen P, Haapiainen R and Kemppainen E: Proinflammatory effects of pancreatic elastase are mediated through TLR4 and NF-kappaB. Biochem Biophys Res Commun 323: 192-196, 2004.

15. Li Y, Zhou ZG, Zhang J, Chen YD, Li HG, Gao HK, Wang R and $\mathrm{Hu}$ TZ: Microcirculatory detection of Toll-like receptor 4 in rat pancreas and intestine. Clin Hemorheol Microcirc 34: 213-219, 2006.

16. Watanabe T, Kudo M and Strober W: Immunopathogenesis of pancreatitis. Mucosal Immunol 10: 283-298, 2017.

17. Zhou R, Yazdi AS, Menu P and Tschopp J: A role for mitochondria in NLRP3 inflammasome activation. Nature 469: 221-225, 2011.

18. Cai X, Chen J, Xu H, Liu S, Jiang QX, Halfmann R and Chen ZJ: Prion-like polymerization underlies signal transduction in antiviral immune defense and inflammasome activation. Cell 156: 1207-1222, 2014.

19. Jin HZ, Yang XJ, Zhao KL, Mei FC, Zhou Y, You YD and Wang WX: Apocynin alleviates lung injury by suppressing NLRP3 inflammasome activation and NF- $\kappa B$ signaling in acute pancreatitis. Int Immunopharmacol 75: 105821, 2019.

20. Baroja-Mazo A, Martín-Sánchez F, Gomez AI, Martínez CM, Amores-Iniesta J, Compan V, Barberà-Cremades M, Yagüe J, Ruiz-Ortiz E, Antón J, et al: The NLRP3 inflammasome is released as a particulate danger signal that amplifies the inflammatory response. Nat Immunol 15: 738-748, 2014.

21. Ma Q, Chen S, Hu Q, Feng H, Zhang JH and Tang J: NLRP3 inflammasome contributes to inflammation after intracerebral hemorrhage. Ann Neurol 75: 209-219, 2014. 
22. Chou RC, Kim ND, Sadik CD, Seung E, Lan Y, Byrne MH, Haribabu B,Iwakura Y andLuster AD: Lipid-cytokine-chemokine cascade drives neutrophil recruitment in a murine model of inflammatory arthritis. Immunity 33: 266-278, 2010.

23. Mayadas TN, Cullere $X$ and Lowell CA: The multifaceted functions of neutrophils. Annu Rev Pathol 9: 181-218, 2014.

24. Kuo YC, Tsai WJ, Meng HC, Chen WP, Yang LY and Lin CY: Immune reponses in human mesangial cells regulated by emodin from Polygonum hypoleucum Ohwi. Life Sci 68: 1271-1286, 2001.

25. Xu C, Zhang J, Liu J, Li Z, Liu Z, Luo Y, Xu Q, Wang M, Zhang G, Wang $\mathrm{F}$ and Chen $\mathrm{H}$ : Proteomic analysis reveals the protective effects of emodin on severe acute pancreatitis induced lung injury by inhibiting neutrophil proteases activity. J Proteomics 220: 103760, 2020.

26. Xu J, Huang B, Wang Y, Tong C, Xie P, Fan R and Gao Z: Emodin ameliorates acute lung injury induced by severe acute pancreatitis through the up-regulated expressions of AQP1 and AQP5 in lung. Clin Exp Pharmacol Physiol 43: 1071-1079, 2016.

27. Wu Y, Tu X, Lin G, Xia H, Huang H, Wan J, Cheng Z, Liu M, Chen $\mathrm{G}$, Zhang $\mathrm{H}$, et al: Emodin-mediated protection from acute myocardial infarction via inhibition of inflammation and apoptosis in local ischemic myocardium. Life Sci 81: 1332-1338, 2007.

28. Ding Y, Zhao L, Mei H, Zhang SL, Huang ZH, Duan YY and Ye P: Exploration of Emodin to treat alpha-naphthylisothiocyanate-induced cholestatic hepatitis via anti-inflammatory pathway. Eur J Pharmacol 590: 377-386, 2008.

29. Gao Z, Sui J, Fan R, Qu W, Dong X and Sun D: Emodin protects against acute pancreatitis-associated lung injury by inhibiting nlpr3 inflammasome activation via Nrf2/HO-1 signaling. Drug Des Devel Ther 14: 1971-1982, 2020.

30. Xu C, Luo Y, Ntim M, Quan W, Li Z, Xu Q, Jiang L, Zhang J, Shang D, Li L, et al: Effect of emodin on long non-coding RNA-mRNA networks in rats with severe acute pancreatitis-induced acute lung injury. J Cell Mol Med 25: 1851-1866, 2021

31. Guan M,MaH,Fan X,Chen X,Miao Mand Wu H: Dexamethasone alleviate allergic airway inflammation in mice by inhibiting the activation of NLRP3 inflammasome. Int Immunopharmacol 78: $106017,2020$.

32. Yoshida K, Okamura H, Hiroshima Y, Abe K, Kido JI, Shinohara Y and Ozaki K: PKR induces the expression of NLRP3 by regulating the NF- $\kappa$ B pathway in Porphyromonas gingivalis-infected osteoblasts. Exp Cell Res 354: 57-64, 2017.

33. Zhang JW, Zhang GX, Chen HL, Liu GL, Owusu L, Wang YX, Wang GY and Xu CM: Therapeutic effect of Qingyi decoction in severe acute pancreatitis-induced intestinal barrier injury. World J Gastroenterol 21: 3537-3546, 2015.

34. Schmidt J, Rattner DW, Lewandrowski K, Compton CC, Mandavilli U, Knoefel WT and Warshaw AL: A better model of acute pancreatitis for evaluating therapy. Ann Surg 215: 44-56, 1992.

35. Wu Q, Gui P, Yao S and Xiang H: Expression of integrin alpha v beta 6 in rats with ventilator-induced lung injury and the attenuating effect of synthesized peptide S247. Med Sci Monit 14: BR41-BR48, 2008.

36. Martinon F, Burns K and Tschopp J: The inflammasome: A molecular platform triggering activation of inflammatory caspases and processing of proIL-beta. Mol Cell 10: 417-426, 2002.

37. Khomtchouk KM, Joseph LI, Khomtchouk BB, Kouhi A, Massa S, Xia A, Koliesnik I, Pletzer D, Bollyky PL and Santa Maria PL: Treatment with a neutrophil elastase inhibitor and ofloxacin reduces P. aeruginosa burden in a mouse model of chronic suppurative otitis media. NPJ Biofilms Microbiomes 7: 31, 2021.

38. Xu Y, Lu B, Zhang N, Liang Y, Gao Y, Ye X and Liu W: Neutrophil extracellular traps are not produced in pediatric patients with one-lung ventilation: A prospective, single-center, observational study. Transl Pediatr 9: 775-783, 2020.
39. Browne GW and Pitchumoni CS: Pathophysiology of pulmonary complications of acute pancreatitis. World J Gastroenterol 12: 7087-7096, 2006.

40. Gao Z, Xu J, Sun D, Zhang R, Liang R, Wang L and Fan R: Traditional Chinese medicine, Qing Ying Tang, ameliorates the severity of acute lung injury induced by severe acute pancreatitis in rats via the upregulation of aquaporin-1. Exp Ther Med 8: 1819-1824, 2014.

41. Zhang H, Neuhöfer P, Song L, Rabe B, Lesina M, Kurkowski MU, Treiber M, Wartmann T, Regnér S, Thorlacius H, et al: IL-6 trans-signaling promotes pancreatitis-associated lung injury and lethality. J Clin Invest 123: 1019-1031, 2013.

42. Janowitz T, Kleeman S and Vonderheide RH: Reconsidering dexamethasone for antiemesis when combining chemotherapy and immunotherapy. Oncologist 26: 269-273, 2021.

43. Pontes-Quero GM, Benito-Garzón L, Pérez Cano J, Aguilar MR and Vázquez-Lasa B: Modulation of inflammatory mediators by polymeric nanoparticles loaded with anti-inflammatory drugs. Pharmaceutics 13: 290, 2021

44. Sugiyama Y, Kato S, Abe M, Mitsufuji S and Takeuchi K: Different effects of dexamethasone and the nitric oxide synthase inhibitor L-NAME on caerulein-induced rat acute pancreatitis, depending on the severity. Inflammopharmacology 13: 291-301, 2005.

45. Buzatto AZ, Malkawi A, Sabi EM, Mujamammi AH, Li L and Abdel Rahman AM: Tissue lipidomic alterations induced by prolonged dexamethasone treatment. J Proteome Res 20: 1558-1570, 2021

46. Grommes J and Soehnlein O: Contribution of neutrophils to acute lung injury. Mol Med 17: 293-307, 2011.

47. Faller S, Hausler F, Goeft A, von Itter MA, Gyllenram V, Hoetzel A and Spassov SG: Hydrogen sulfide limits neutrophil transmigration, inflammation, and oxidative burst in lipopolysaccharide-induced acute lung injury. Sci Rep 8: 14676, 2018.

48. Amaral FA, Costa VV, Tavares LD, Sachs D, Coelho FM, Fagundes CT, Soriani FM, Silveira TN, Cunha LD, Zamboni DS, et al: NLRP3 inflammasome-mediated neutrophil recruitment and hypernociception depend on leukotriene $\mathrm{B}(4)$ in a murine model of gout. Arthritis Rheum 64: 474-484, 2012.

49. Inoue Y, Shirasuna K, Kimura H, Usui F, Kawashima A, Karasawa T, Tago K, Dezaki K, Nishimura S, Sagara J, et al: NLRP3 regulates neutrophil functions and contributes to hepatic ischemia-reperfusion injury independently of inflammasomes. J Immunol 192: 4342-4351, 2014

50. Fann DY, Lee SY, Manzanero S, Chunduri P, Sobey CG and Arumugam TV: Pathogenesis of acute stroke and the role of inflammasomes. Ageing Res Rev 12: 941-966, 2013.

51. Garlanda C, Dinarello CA and Mantovani A: The interleukin-1 family: Back to the future. Immunity 39: 1003-1018, 2013.

52. Crother TR, Porritt RA, Dagvadorj J, Tumurkhuu G, Slepenkin AV, Peterson EM, Chen S, Shimada K and Arditi M: Autophagy limits inflammasome during chlamydia pneumoniae infection. Front Immunol 10: 754, 2019.

53. Sun Y, Abbondante S, Karmakar M, de Jesus Carrion S, Che C, Hise AG and Pearlman E: Neutrophil caspase-11 is required for cleavage of caspase- 1 and secretion of IL-1 $\beta$ in aspergillus fumigatus infection. J Immunol 201: 2767-2775, 2018.

54. McDonald B, Pittman K, Menezes GB, Hirota SA, Slaba I, Waterhouse CC, Beck PL, Muruve DA and Kubes P: Intravascular danger signals guide neutrophils to sites of sterile inflammation. Science 330: 362-366, 2010.

55. Zhou W, McCollum MO, Levine BA and Olson MS: Role of platelet-activating factor in pancreatitis-associated acute lung injury in the rat. Am J Pathol 140: 971-979, 1992.

This work is licensed under a Creative Commons Attribution-NonCommercial-NoDerivatives 4.0 International (CC BY-NC-ND 4.0) License. 